\title{
An Experimental Program in Neutrinos, Nucleon Decay and Astroparticle Physics Enabled by the Fermilab Long-Baseline Neutrino Facility
}

\author{
Alec Habig ${ }^{* \dagger}$ \\ University of Minnesota Duluth \\ E-mail: ahabig@umn.edu
}

\begin{abstract}
A new International Team (DUNE - Deep Underground Neutrino Experiment) has been formed to pursue an accelerator-based long-baseline neutrino experiment, as well as neutrino astrophysics and nucleon decay, with an approximately $40 \mathrm{kt}$ (fiducial) modular liquid argon TPC (LAr-TPC) detector located deep underground and a high-resolution near detector. Several independent worldwide efforts, developed through years of detailed studies, are converging around the opportunity provided by the megawatt neutrino beam facility planned at Fermilab and by the new significant expansion with improved access at the Sanford Underground Research Facility in South Dakota, 1,300 km from Fermilab.

The principle goals of this experiment are: a comprehensive investigation of neutrino oscillations to test $\mathrm{CP}$ violation in the lepton sector, determine the ordering of the neutrino masses, and test the three-neutrino paradigm; to perform a broad set of neutrino scattering measurements with the near detector; and to exploit the large, high-resolution, underground far detector for non-accelerator physics topics including atmospheric neutrino measurements, searches for nucleon decay, and measurement of astrophysical neutrinos especially those from a core-collapse supernova.
\end{abstract}

The European Physical Society Conference on High Energy Physics

22-29 July 2015

Vienna, Austria

${ }^{*}$ Speaker.

${ }^{\dagger}$ for the DUNE collaboration 


\section{Organization}

The Deep Underground Neutrino Experiment (DUNE) is the new name for the combined efforts of the collaborations formerly known as LBNE and LBNO with some new participants. It has new leadership: Mark Thomsen of Cambridge and André Rubbia of ETH Zurich are the spokesmen; a new Conceptual Design Report [1]; and has passed the US Department of Energy's "CD1" milestone in July 2015. DUNE covers the detector and science goals of the project: the beam and infrastructure part of the project is now called the Long-Baseline Neutrino Facility (LBNF). The DUNE collaboration includes 750 scientists from 150 institutions in 23 countries. The goals this collaboration is working towards are described in this talk, with details of all sorts available online in the CDR [1].

\section{Experimental Overview}

DUNE aims to study neutrino oscillations using a new more intense beam of $v_{\mu}$ from Fermilab to an underground $40 \mathrm{kt}$ liquid argon Time Projection Chamber (TPC) on the 4850 level of the Sanford Underground Lab. The 1,300 km baseline will be longer and cross more mass than current beams do, allowing for a better probe of matter effects in $v_{\mu} \rightarrow v_{e}$ appearance and thus mass hierarchy and $\delta_{C P}$. The new beam will be $1.2 \mathrm{MW}$, increasing the statistics by creating more neutrino interactions in the far detector; and tunable in energy, allowing more parameter space to be mapped out. A liquid argon TPC has fine spatial resolution, making the most out of each individual neutrino interaction and decreasing systematic error bars. The LArTPC modules will be staged as four separate $10 \mathrm{kt}$ modules, the first of which will be a single phase detector in the mold of ICARUS, with the possibility of later modules to be dual-phase designs to catch ionization in the gas above the liquid. Both methods are the subject of intense R\&D, with LARIAT, MicroBoone, CAPTAIN, and SBND exploring single-phase issues at Fermilab; and WA105 prototyping the dual-phase methods at CERN.

To best evaluate the neutrino signal seen at the "far detector" at Homestake, a "near detector" near the beam source at Fermilab is needed to precisely measure the pre-oscillation neutrino beam and thus establish a precise "before and after" measurement. The reference design for this instrument is a fine-grained straw tube tracker with calorimeters and a $0.6 \mathrm{~T}$ magnet. The near detector will also be able to study the neutrino interactions themselves, expanding our knowledge of how neutrinos interact.

\section{Beam Neutrino Physics Goals}

The parameters of the beam and detectors being designed are set by the desire to be sensitive to as many possible different values of the currently unknown parameter $\delta_{C P}$ (the charge-parity violating phase of a neutrino) and learn what the neutrino mass hierarchy might be for any given value of $\delta_{C P}$. Figure 1 shows the reach of DUNE for these parameters vs. exposure: $5 \sigma$ sensitivity to the neutrino mass hierarchy for all possible values of $\delta_{C P}$ after $400 \mathrm{kt}-\mathrm{MW}$-years, and $3 \sigma$ sensitivity to $\delta_{C P}$ itself for $75 \%$ of possible values of $\delta_{C P}$ after $850-1300 \mathrm{kt}-\mathrm{MW}$-years. Those are large exposures, but as seen in the figure, the simple act of designing a tunable energy beam (part of the "optimized design") substantially accelerates the physics reach. 

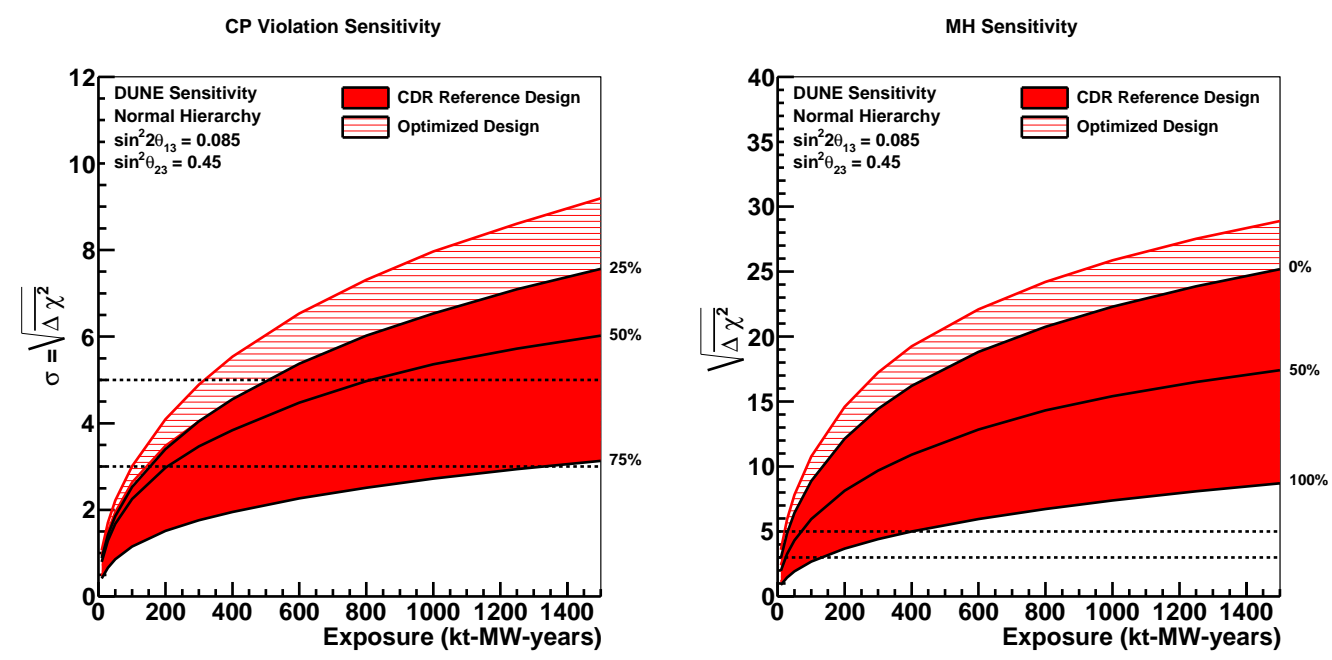

Figure 1: Sensitivities of the DUNE experiment (in $\sigma$ ) to discovery of the CP-violating phase $\delta_{C P}$ (left) and the neutrino mass hierarchy (right). The solid red regions cover the range of possible values of $\delta_{C P}$ (in percent, labeled on the right-hand y-axis). So, for example, the easiest $25 \%$ of potential $\delta_{C P}$ 's to find (the top of the left-hand red region on the left) would be seen at $3 \sigma$ before $400 \mathrm{kt}-\mathrm{MW}$-years of exposure was collected and at $5 \sigma$ before $400 \mathrm{kt}-\mathrm{MW}$-years was in the bag. However, the hardest $25 \%$ (left after the labeled $75 \%$ could be seen at $1300 \mathrm{kt}-\mathrm{MW}$-years) would remain unresolvable. The hashed area represents the improvement to be gained by optimizing the beam design and represents where the top of the red region would lie with a tunable beam

These parameters are extracted from the data by looking at the oscillatory patterns in the neutrino appearance probability. While the overall probability of $v_{e}$ appearing from a beam of pure $\nu_{\mu}$ is governed by the recently discovered $\theta_{13}$ parameter, the presence of MSW-style matter effects on the oscillation as the beam passes through the earth impose a different structure on the spectrum of appearing $v_{e}$ compared to that of $\bar{v}_{e}$ in an anti-neutrino beam: more matter in the baseline creates a larger effect, driving the location of the DUNE far detector. A "Normal Hierarchy" where the solar doublet of mass eigenstates is larger than the third state would experience an enhancement of the $v_{\mu} \rightarrow v_{e}$ appearance caused by coherent forward scattering of $v-e$ on electrons in the earth: the "Inverted Hierarchy" would likewise experience a reduction in the appearance oscillations. This experiment's goal is to be able to tell apart the patterns shown in Fig. 2: hence the need for a LArTPC's fine energy resolution and sensitivity to the resulting electromagnetic shower, and the need for long exposures to both neutrino and anti-neutrino beams.

In addition to neutrinos from the intense (but comparatively narrow-band) beam, the DUNE far detector would be sensitive to neutrinos coming from cosmic ray interactions around the world. These atmospheric neutrinos probe a wide range of oscillations parameter space that is complementary to the beam: and they are measured in the same detector as the beam neutrinos, minimizing the systematic uncertainty present when comparing neutrino datasets from different experiments.

\section{Other Physics Goals}

Such a large, high resolution detector, sited deep underground to reduce cosmic ray and cos- 

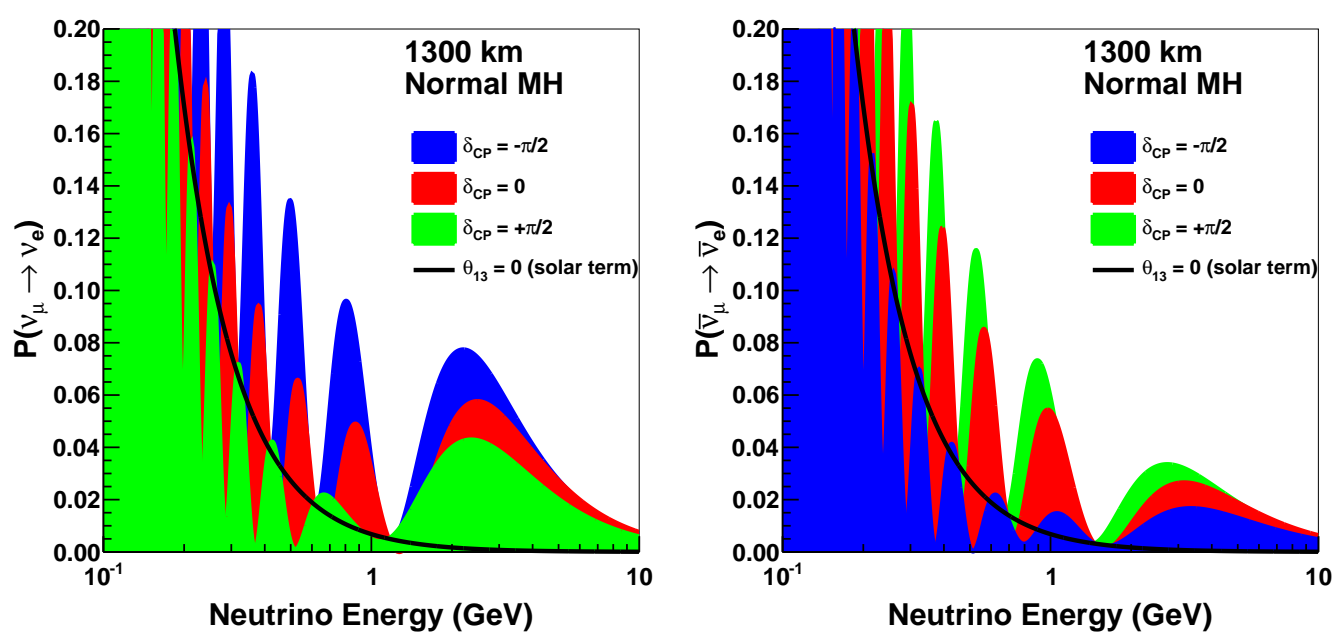

Figure 2: $v_{e}$ appearance spectra for a $1300 \mathrm{~km}$ baseline beam of $v_{\mu}$ (left) and $\bar{v}_{\mu}$ (right), for different values of $\delta_{C P}$.

mogenic backgrounds, is well-suited to push the bounds of a number of other areas of research. One such topic is the search for proton decay, particularly modes involving kaons, predicted by many super-symmetric models. LArTPC's spatial resolution and low thresholds are ideal for identifying the outgoing kaon, and $40 \mathrm{kt}$ of mass operating for years would push limits far past what current detectors could do.

Neutrinos make up $99 \%$ of the energy released when a large star reaches the end of its life and undergoes core collapse, creating a supernova. While this basic scheme was confirmed by observations of neutrinos from SN1987A, a supernova somewhere in our galaxy as observed by the DUNE detector would generate an order of magnitude more interactions. Liquid Argon is sensitive to electron neutrinos and neutral current interactions: other water-based or scintillatorbased neutrino experiments are primary sensitive to anti-electron neutrinos. Observing the time evolution of the neutrino "light curves" will be an unprecedented window into one of the most extreme places in the astrophysical universe, and seeing what those high densities of neutrinos and matter do to the neutrinos themselves via extreme matter effects and neutrino collective interactions that are not possible to probe in a lab.

As well as providing a "before" picture to the "after" oscillations spectra seen in the far detector, the intense beam as seen in the DUNE near detector will provide high statistics insights into the way neutrinos interact with matter.

Additionally, current neutrino experiments have taught us that it is possible to use the detectors for many other analyses. For example the atmospheric neutrinos can also be used for astrophysical neutrino observations and indirect dark matter searches. Many models of Lorentz or CPT violation, non-standard interactions, and sterile neutrinos would impose variations on the beam neutrinos which could be seen. The cosmic ray backgrounds to these neutrino searches are themselves of interest to cosmic ray physicists. Finally, if backgrounds can be controlled well enough to push down energy thresholds, DUNE might also be able to study solar neutrinos or the diffuse supernova neutrino background. 


\section{Schedule and Conclusion}

The task of building this large experiment is daunting and expensive, and then it must run in the beam for many years to reach the science goals outlined above. Thus, work is happening now on design and prototyping, with civil construction and excavating planned for FY18 and a start on the far detector at Homestake planned for FY20. The first LArTPC module will be commissioned in FY25 with the beamline completed in FY26.

The Deep Underground Neutrino Experiment is a large experiment with large goals, and will take a lot of work over many years to come to fruition. However, that process is well under way, has many collaborators and a solid funding plan, and is off to a good start.

\section{References}

[1] "LBNF/DUNE Conceptual Design Report", https : / / web. fnal.gov/project/LBNF / ReviewsAndAssessments/LBNF_DUNE20DOE20CD-1\%20Refresh\%20Review/ SitePages/Conceptual $\div 20$ Design 20 Report. aspx, and references therein, 2015. Headed to an arXiv listing soon for easier reference 